\title{
The Space Shuttle Columbia Accident Investigation and Reconstruction: Two Years Later
}

\author{
S. J. McDanels, ${ }^{*}$ Richard W. Russell**
}

*Chief, Failure Analysis and Materials Evaluation Branch, YA-F1, NASA, Kennedy Space Center, FL, 32899

** Aging Aircraft Principal Engineer, NASA Orbiter Project Office, MV7, Kennedy Space Center, FL, 32899

The Space Shuttle Columbia was lost during re-entry over two years ago. Since the release of the official materials-related findings in August of 2003, additional testing and analysis of select pieces of debris has continued. Microanalytical techniques, including EMPA, ESCA, and x-ray elemental dot mapping, were employed during the initial investigation; the results related the microstructural characteristics of deposit layers to the breach location in the leading edge of the left wing. Such characteristics included deposition order, composition, and distribution. Subsequent to the original efforts, new analytical data and information, not available at the time of the primary investigation, has been generated. This data was obtained via a low-vacuum SEM, fitted not only with a lightelement EDS detector, but an XRF tube as well. Essentially, for elements up to sodium, classic EDS was utilized; above sodium, XRF was used. Predominantly, the elements of interest were aluminum, titanium, chromium, iron, nickel, and copper. The findings of both old and new data are compared, and their application to the overall accident investigation detailed.

During the initial stages of the investigation, samples as shown in Fig. 1 were harvested from selected pieces of debris and subjected to further examination. Ultimately, the information derived from the sampling and analysis was used to determine the most likely location of a breach in the left wing leading edge of the Columbia, as shown in Fig. 2. Similarly, the information was used to determine the path which the impinging plasma followed once inside the wing structure.

Now, two years later, additional inspection criteria have been instituted, new launch rules have been introduced, and more demanding restrictions put in place to minimize potential FOD damage to the vehicle and the likelihood of foam loss from the external tank. Likewise, strategies such as on-orbit inspection and repair of thermal protection system components are currently being developed and should be implemented by the return to flight. Other post-flight inspections, including in-situ thermography, ultrasound, and eddy current evaluation, are being investigated to also help ensure the improved safety of the fleet. 

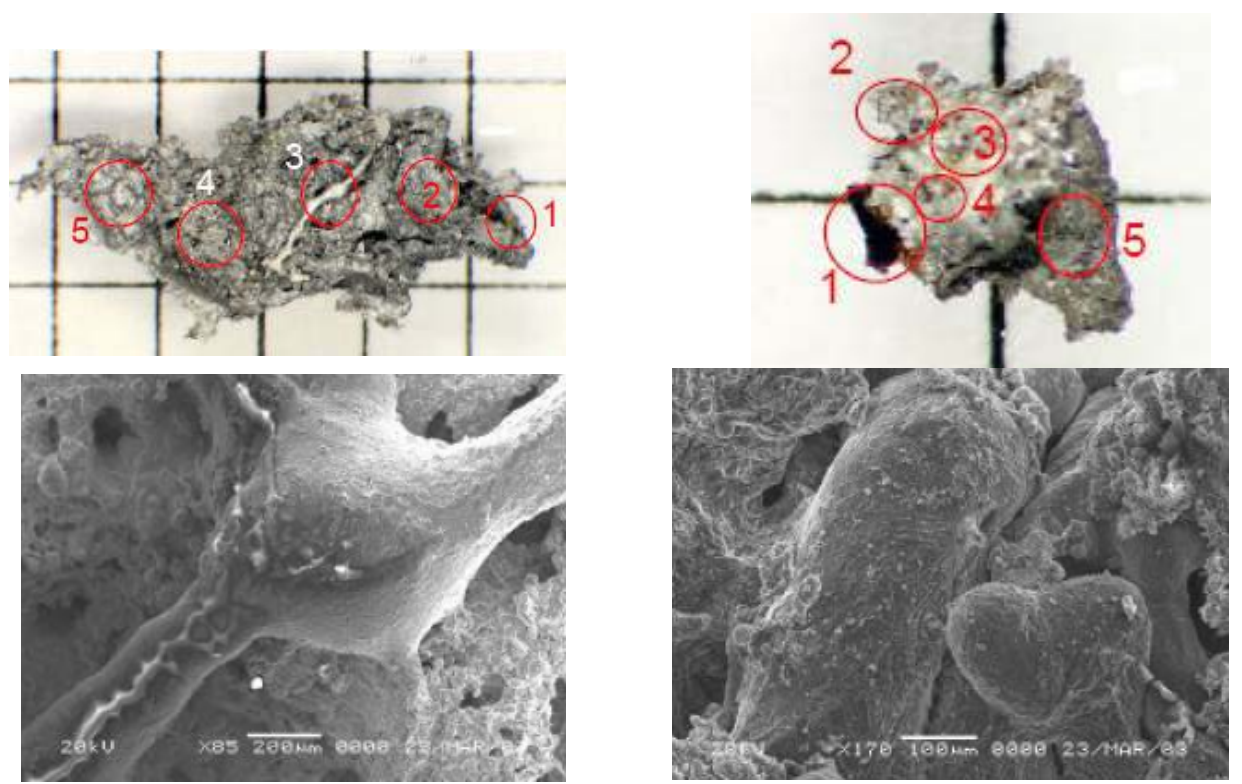

FIG. 1. Macroscopic views of typical slag samples (magnification of top photos approximately 5X) and scanning electron micrographs (magnification of bottom photos $85 \mathrm{X}$ (left) and 170X (right)) of selected areas.

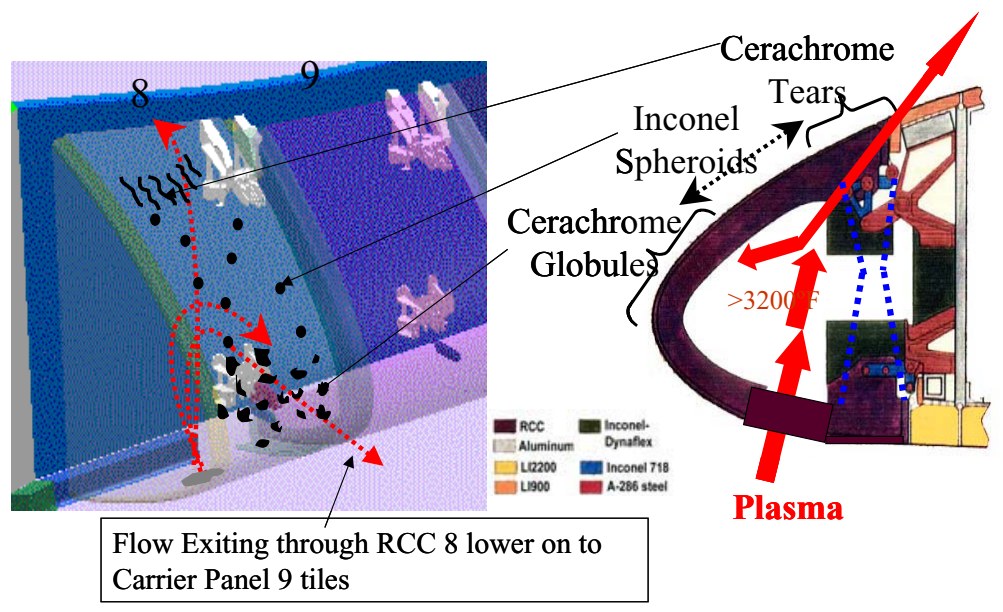

FIG. 2. Schematic representation of the suspected breach location and plasma flow path. Taken from the STS-107 Columbia Reconstruction Report, NSTS-60501, National Aeronautics and Space Administration, June, 2003. 